\title{
La enseñanza de la historia en el currículum chileno de educación básica como reflejo del contexto político actual ${ }^{1}$
}

\section{KEY WORDS}

history teaching, curriculum, cultural materialism, social movements

\begin{abstract}
Sáez-Rosenkranz Isidora, La enseñanza de la historia en el currículum chileno de educación básica como reflejo contexto político actual [History Teaching in the Chilean Curriculum on Primary Education as a Reflect of the Current Political Context]. Kultura - Społeczeństwo Edukacja nr 2(10) 2016, Poznań 2016, pp. 9-22, Adam Mickiewicz University Press. ISSN 2300-0422. DOI 10.14746/kse.2016.9.1.

The aim of this article is to explain the characteristics on current History teaching proposed into the curriculum of primary education in Chile under the present social process. We employ Raymond Williams cultural materialism and specifically his categories on cultural products to apprehend the curriculum and link it to the context where it is pro duced. To do so, we use the historical method, and considering pedagogical and didactical elements. The documental corpus analyzed is conformed by the official documents guiding education but also other historical sources coming from the current political situation. The results on this analysis show that there is a curricular dichotomy between traditional history teaching based on events of national history and learning by rote and, current didactical proposals, which tend to develop historical thinking. This situation reflects the political tension on social demands and the institutional longstanding objectives for education.
\end{abstract}

\section{Introducción}

Actualmente Chile vive un proceso de reforma curricular a causa de la promulgación de la Ley General de Educación en el año 2009 (en adelante LGE). Este cambio normativo fue producto de una intensa movilización estudiantil,

\footnotetext{
${ }^{1}$ Este artículo deriva de la tesis doctoral "Análisis de actividades en libros de texto de Historia, Geografía y Ciencias Sociales de educación básica en Chile" llevada a cabo en la Universidad de Barcelona mediante la beca APIF y ha sido dirigida por el Dr. Joaquín Prats Cuevas.
} 
aun en desarrollo, que tuvo como principal hito la Revolución Pingüina ${ }^{2}$ del año 2006. Allí, los estudiantes de educación secundaria obligatoria cuestionaron la ley heredada de la Dictadura Militar (LOCE, 1990-2009) que tenía un marcado carácter nacionalista y neoliberal (Cabalin, 2012; Gazmuri Stein, 2013).

Como consecuencia del cambio legislativo fue necesario reestructurar el currículum. En 2012 comenzó una reforma que en la práctica aplicó la reestructuración de la escolaridad obligatoria establecida en la LGE (6 años de educación básica, cuatro de educación media y dos de educación diferenciada), a la vez que consolidó la enseñanza de la Historia en asignatura de Historia, Geografía y Ciencias Sociales en toda la educación obligatoria ${ }^{3}$.

El nuevo currículum de educación básica se organizó en torno a objetivos de aprendizajes de dos tipos, habilidades y ejes disciplinares. Las habilidades se plantean en cuatro ámbitos: pensamiento espacial y temporal, análisis y trabajo con fuentes, pensamiento crítico y comunicación. Los ejes disciplinares son tres, Historia, Geografía y Formación Ciudadana.

Esta nueva organización pretende que los estudiantes "adquieran un sentido de identidad y pertenencia a la sociedad" mediante el desarrollo de "conocimientos, habilidades y actitudes necesarias para comprender la compleja realidad social contemporánea y su devenir, para desenvolverse como un ciudadano capaz de actuar responsable y críticamente en la sociedad, y para enfrentar los desafíos de un mundo globalizado"(Ministerio de Educación, 2012: 178).

Este planteamiento contrasta con el objetivo de la enseñanza de la Historia propuesto en la LOCE, donde se señalaba que se pretendía "Desarrollar su sentido patrio y conocer la historia y geografía de Chile con la profundidad" (Ministerio de Ministerio de Educación, 1990: Artículo 11, letra c.) A partir de la diferencia de perspectiva entre uno y otro documento, el objetivo de este artículo es explicar los discursos sobre la enseñanza de la Historia en educación básica. Esto se realiza a partir del análisis del currículum concebido como materialidad. De forma específica, se analizan la parte introductoria y la referida a Historia desde un punto de vista didáctico y pedagógico y en relación al contexto social en el que este nuevo marco normativo se gestó.

\footnotetext{
${ }^{2}$ Se denominó Revolución Pingüina por que las estudiantes de educación secundaria de centros públicos suelen usar un uniforme escolar azul oscuro y camisa blanca que se asemeja al aspecto de un pingüino. A causa de este uniforme, coloquialmente se les denomina "pingüinos" a los estudiantes de la educación obligatoria.

${ }^{3}$ En el currículum anterior la asignatura se estructuró de la siguiente manera: $1^{\circ}$ a $4^{\circ}$ básico, una asignatura que integró Ciencias Naturales e Historia (Estudio y comprensión del medio natural, social y cultural), donde el único contenido de Historia eran los pueblos precolombinos. (Toledo Jofré \& Renato Gazmuri Stein, 2009). De $5^{\circ}$ a $8^{\circ}$ básico, una materia que apuntaba al estudio de la Historia y las Ciencias Sociales (Estudio y comprensión de la sociedad) y $1^{\circ}$ a $4^{\circ}$ medio, la asignatura de Historia, Geografía y Ciencias Sociales.
} 


\section{Metodología}

Para analizar el currículum en relación al contexto en el que se produce, se ha empleado la metodología histórica (Sáez-Rosenkranz, 2016) y un análisis didáctico y pedagógico para valorar los aprendizajes promovidos en el documento técnico. En el caso de los pedagógicos se empleó la taxonomía de Anderson y Krathwohl (Anderson \& Krathwohl, 2001). Para el caso de la didáctica de la Historia se puso énfasis en los aspectos característicos del pensamiento histórico (Seixas \& Morton, 2013).

En cuanto a la metodología histórica, el corpus documental empleado está conformado por el currículum (Bases curriculares de educación básica y LGE), así como otros documentos que permiten recoger información sorbe su construcción, como las discusiones en el parlamento o los informes de comisiones. En el caso del análisis didáctico y pedagógico se aplica en particular a las bases curriculares, comparando lo declarado en las secciones introductorias y los objetivos de aprendizaje, bajo las categorías de tipo de conocimiento y acción cognitiva (Anderson \& Krathwohl, 2001). Aquí se consideraron los verbos de aprendizaje y se clasificaron los tipos de contenidos. En el caso de las cuestiones didácticas, se han seguido las directrices del pensamiento histórico propuestas desde la didáctica de la Historia (Prats, 2011; Seixas \& Morton, 2013) que señalan como aprendizajes relevantes para la formación de sujetos críticos, la aplicación de procedimientos propios de la Historia, así como la adquisición de aprendizajes complejos como el pensamiento temporal, el uso de evidencias, la empatía y el juicio histórico.

\section{La enseñanza de la Historia desde la didáctica de las ciencias sociales}

Desde hace ya tres décadas, la Didáctica de la Historia ha propuesto una enseñanza no solo basada en el conocimiento de los grandes acontecimientos y personajes de las historias nacionales, sino a promover lo que se denomina pensamiento histórico (Carretero, 2011; Seixas \& Morton, 2013; Wineburg, 2001). Esto implica propender aprendizajes que van más allá de conocimientos declarativos de primer orden (el qué, cuándo o quién ha llevado a cabo las distintas acciones bajo un relato secuencial), sino que poner énfasis también en aprendizajes de segundo orden y procedimientos. Los conocimientos de segundo orden, según Trepat (1995), constituyen aprendizajes intermedios entre los hechos factuales y los procedimientos. Aquí situamos la adquisición de conceptos claves como el trabajo del tiempo del modo que lo hace el historiador, el énfasis 
en las causalidades y consecuencias, el desarrollo de la empatía y juicio histórico como la articulación de valoraciones fundamentadas en evidencias (Prats, 2011; Seixas \& Morton, 2013). Además se propone trabajar en aula empleando el método histórico basado en la formulación de hipótesis y su comprobación o refutación mediante la investigación con fuentes históricas. Procedimientos claves son la crítica, lectura, clasificación y análisis de fuentes (Prats, 2011). Además aparece como elemento importante la capacidad de articular relatos explicativos sobre los fenómenos o narrativa histórica (Rüsen, 2004).

Estas ideas son centrales a la hora de desarrollar el pensamiento histórico, el cual se logra mediante una adecuada programación de aula como lo han demostrado experiencias didácticas de este tipo (Salazar-Jiménez, Barriga-Ubed, \& Ametller-López, 2015). Así en los estudiantes se promueve la capacidad de enfrentar su presente de forma crítica y poder actuar en su entorno (Prats, 2011).

Ciertamente esta propuesta ha calado poco en las formas de enseñanza que se llevan a cabo en el aula, lo que se ha demostrado a través de los análisis de los libros de texto (Sáiz Serrano, 2013) y la percepción que tienen los estudiantes respecto a la asignatura. Los estudios tienden a señalar que la Historia sirve para conocer la historia del país (Fink, 2005). Esto tiene relación con las perspectivas curriculares bajo las cuales se ha propuesto el aprendizaje de la Historia, ya que se ha orientado a promover metodologías dirigidas a la repetición de acontecimientos en lugar del análisis de fenómenos sociales (Bellatti, Gámez Ceruelo, \& Trepat Carbonell, 2015; Toledo Jofré \& Renato Gazmuri Stein, 2009).

\section{Los currículum como producto cultural}

Abordar desde una perspectiva crítica y explicativa los aprendizajes en Historia propuestos en el currículum, implica no solo conocer qué tipo de enseñanza se promueve, sino también aprehenderlo desde un enfoque diferente. En este caso nos aproximamos al currículum desde el materialismo cultural y lo concebimos como un producto cultural más allá de su dimensión educativa. Adherimos a la idea que los elementos que conforman la cultura son los órdenes sociales que la producen en conjunto con las prácticas culturales y sus productos (Williams, 1981). Nos valemos de esta perspectiva, y en particular de los aportes de Raymond Williams $(1981,1983,2000)$ para aprehender ontológicamente los currículums y sus aprendizajes promovidos, puesto que los asumimos como productos culturales. Bajo esta teoría, lo cultural y su materialidad son aspectos constitutivos de la sociedad, por lo tanto representan una parte ella. En su proceso de producción existen diversos agentes - entre los que encontramos las instituciones - y fuerzas en tensión que les dan forma: la hegemonía y la contrahegemonía. 
Al llevar esto al campo educativo, y a nuestro tema en particular, vemos que nos concentramos en el ámbito hegemónico, dominante, ya que es aquí donde se gestan. Sin embargo, su uso y la aplicación práctica que hacen de ellos los profesores se sitúa en el opuesto, en un lugar contrahegemónico. Allí hay construcciones simbólicas de los sujetos que las recepcionan (los profesores y estudiantes), que pueden interpretar y aplicar los lineamientos de una forma diferente a como desde la institucionalidad son producidos ${ }^{4}$.

Para explicar las dinámicas de la producción cultural de una determinada sociedad, Williams acuñó el concepto de estructura de sentir o estructura de sentimiento, que las concibe como inacabadas. Los productos culturales emergen en un determinado contexto y son recepcionados en otro, igualmente específico, pero en el cual la carga histórica de los sujetos que los producen e intervienen, los codifica y decodifican de modos determinados (Williams, 2000). Siguiendo esta propuesta teórica, en este trabajo concebimos los productos materiales y simbólicos de los sistemas educativos, en este caso el currículum, como productos culturales, puesto que son fruto - visible y tangible - de ciertos ordenes sociales y culturales ${ }^{5}$.

La importancia de reflexionar y plantear estas cuestiones deriva de las implicancias sociales que se encuentran en los discursos que articula el currículum y otros recursos escolares institucionales. Allí finalmente adquieren una materialidad que opera en el cotidiano de los sistemas educativos.

A su vez, Williams sostiene que en los productos culturales convergen tres tipos de fuerzas, las dominantes, las residuales y las emergentes. Las primeras, marcadas por los espacios hegemónicos, las segundas por aquellos elementos históricos, casi tradicionales y, las terceras, vinculadas a cuestiones circunstanciales que aparecen en determinados momentos con causas específicas (1981). Por tanto, en el abordaje del currículum y su relación con el contexto en que se producen, nos valemos de esta perspectiva teórica y sus características.

\section{Los movimientos sociales, la crisis de la institucionalidad chilena y el cambio curricular}

El advenimiento de la dictadura militar (1973-1989) rompió el modelo de desarrollo hacia adentro que se venía dando desde la década del ' 40 , el cual con-

\footnotetext{
${ }^{4}$ Aquí consideramos importante la posibilidad de actitud crítica que tienen los profesores respecto a los materiales que les son dados, la flexibilidad de seleccionar los recursos del libro de texto que les son útiles, y las estrategias didácticas que empleen mas allá de las sugerencias otorgadas por las guías para el docente.

${ }^{5}$ Sobre esta discusión pueden revisarse su las obras de Raymond Williams (2000,1981 y 1983).
} 
sideraba al Estado como garante de la educación. La instalación del neoliberalismo, la generación de una nueva constitución en la cual se coartó la participación de la sociedad civil y la promulgación de la LOCE, reorganizaron la estructura institucional y educativa desvinculando, en la práctica, el papel educativo fundamental que el Estado había tenido.

Estas herramientas institucionales promovieron la creación de centros educativos de carácter privado y particulares subvencionados (privados con financiación estatal). Además, la LOCE otorgó a los municipios la responsabilidad de los centros de educación básica y media y dio todas las facilidades para la creación universidades de carácter privado (Donoso, 2004). De esta manera, aumentaba la cobertura de la educación pero con lógicas de mercado que redujeron sustancialmente la matrícula en centros públicos, ampliando a mas de un $60 \%$ la del sector privado con y sin subvención estatal ${ }^{6}$.

A comienzos del siglo XXI aparecieron algunos movimientos estudiantiles, especialmente universitarios, que conscientes del origen institucional del sistema educativo comenzaron una serie de acciones fragmentadas que resonaron en la sociedad civil como reclamos inconsistentes de una juventud revoltosa. Mas, con el pasar de los años, estas reflexiones se fueron acrisolando en voces mas claras. En mayo de 2006, los estudiantes secundarios comenzaron con gran fuerza la citada Revolución Pingüina. Esta puso sobre el tapete de manera estructurada y orgánica el tema de la educación de mercado, la mala calidad de enseñanza en la educación básica y media y cuestionó la LOCE como pilar de esta problemática. Como consecuencia, se logró entablar una mesa de diálogo con el Gobierno el cual convocó al Consejo Asesor Presidencial para la Calidad de Educación, entre cuyos integrantes se contaban parlamentarios, especialistas en educación y miembros de la sociedad civil. Estas instancias, producto de las demandas sociales acabaron por derogar la Ley de Enseñanza dictatorial con la promulgación de la Ley General de Educación (Carrasco Segura, 2007).

Las dinámicas políticas diluyeron las movilizaciones y la llegada al poder de Sebastián Piñera (2010-2014), que materializó en su figura los principios del neoliberalismo impuesto por la dictadura, fue el puntapié inicial para la reemergencia de una serie de movimientos ciudadanos que han puesto en jaque los frutos políticos del régimen militar. Sacaron a las calles a miles de chilenos a protestar y a manifestarse por temas que se discutían normalmente puertas adentro.

En este contexto apareció con mas fuerza en 2011 un movimiento estudiantil que no solo cuestionaba los resultados insatisfactorios de las movilizaciones

\footnotetext{
${ }^{6}$ En Chile un $90 \%$ de los centros educativos privados reciben algún tipo de subvención estatal (Zúñiga-Peña, 2015)
} 
anteriores, sino también planteó propuestas concretas desde tres colectivos vinculados a la educación: estudiantes secundarios, estudiantes universitarios y Colegio de Profesores de Chile. La bandera de lucha fue la misma: sistema nacional de educación pública, gratuita, democrática y de calidad; organizado y financiado por el Estado en todos sus niveles y que en el caso específico de cada sector, se ha materializado en demandas específicas.

El movimiento estudiantil desde 2006 ha contado con el apoyo de distintas entidades institucionales y no institucionales, además de la sociedad civil. A causa de lo anterior, algunos estudios provenientes desde la sociología han señalado que en Chile el modelo neoliberal se está resquebrajando, no solo a partir de la situación de la educación, sino de una serie de sucesos ciudadanos que han devenido de un malestar generalizado (Mayol, 2013).

Es justamente producto de este contexto que se gesta el nuevo currículum, el cual se ve directamente influido por varias razones. En primer lugar, porque su construcción es impulsada por el Ministerio de Educación, institución que ha visto permeado su ámbito de operación por las acciones ciudadanas y los cambios legislativos. En segundo lugar, quienes hacen estos cambios y materialidades son personas, sujetos que definen y construyen el currículum. Ellos están inevitablemente afectados por los vaivenes y discursos del entorno político y la sociedad. En tercer lugar, cada administración con sus sujetos y sus concepciones sobre la educación, plasman en diversos espacios sus ideas. Considerar estos elementos es importante para entender el proceso educativo actual.

\section{Las contradicciones discursivas en el currículum de Historia, Geografía y Ciencias Sociales}

El análisis de los contenidos del currículum de Historia, Geografía y Ciencias Sociales en educación básica bajo los aspectos pedagógicos y didácticos indican que existe una discordancia entre lo declarado en su parte introductoria y el tipo de aprendizaje promovido en los Objetivos de Aprendizaje (en adelante OA).

En la primera parte se señala que se espera que el estudiante mediante la asignatura de Historia alcance "una mejor comprensión de su sociedad y su rol en ella" (Ministerio de Educación, 2012: 178). Así se sostiene que se propende que el educando comprenda, mediante el desarrollo del pensamiento histórico, "que la experiencia de vivir en sociedad está contextualizada en el tiempo" y "la comprensión del presente solo es posible si se examina el pasado y los procesos históricos que han modelado la realidad actual, en la que se reflejan las permanencias y los cambios entre el ayer y e hoy" (Ministerio de Educación, 2012: 178 y 179). Es por ello que "se busca evitar una visión de la historia como 
flujo de fuerzas impersonales y ajenas; por el contrario, se resalta que los estudiantes, en tanto personas y ciudadanos, quienes participan en el devenir histórico de la sociedad" (Ministerio de Educación, 2012: 179).

Por el contrario, los OA planteados para esta asignatura, emplean en su mayoría en todos los cursos, verbos y tipo de conocimiento correspondientes a los niveles más bajos de la taxonomía de Anderson y Krathwohl asociados a los aprendizajes de primer orden. Así por ejemplo se señala en primero básico que uno de los OA es "Conocer sobre la vida de hombres y mujeres que han contribuido a la sociedad chilena en diversos ámbitos" (Ministerio de Educación, 2012: 193). En quinto básico, los lineamientos son semejantes, ya que se indica, por ejemplo, que los estudiantes deben ser capaces de "describir el proceso de conquista de américa y de Chile, incluyendo a los principales actores (...), algunas expediciones y conflictos bélicos (...)” (Ministerio de Educación, 2012: 210).

Como se observa en estos ejemplos, y sucede con la gran mayoría de los OA a lo largo de la educación básica, los verbos remiten a acciones como conocer, explicar y describir y en muy escasa oportunidades a otros más complejos como analizar o hipotetizar. Como en los ejemplos anteriores, el orden de los contenidos tienden a ser los acontecimientos propios de la Historia patria, aunque se han incluido también otros que difieren de estos contenidos históricos. Aquí situamos por ejemplo el siguiente OA de segundo básico en el que se señala que los estudiantes deben "comparar el modo de vida y expresiones culturales de los pueblos indígenas presentes en el Chile actual (...) con respecto al periodo precolombino, identificando aspectos de su cultura que se han mantenido hasta el presente y aspectos que han cambiado" (Ministerio de Educación, 2012: 197). En sexto básico se establece que al término del curso escolar los educandos deben ser capaces de "explicar y dar ejemplos de la progresiva democratización de la sociedad durante el siglo XX, considerando el acceso creciente al voto, la participación de la mujer en la vida pública y el acceso a la educación y a la cultura, entre otros" (Ministerio de Educación, 2012: 216).

Se puede observar a partir de esto fragmentos que en el currículum convergen dos perspectivas de enseñanza de la Historia. Por un lado, la tradicional, mayoritaria, basada en el conocimiento de grandes personajes y hechos de la historia patria, empleando verbos que apuntan a una actitud pasiva del estudiantado. Por otro lado, otros cercanos a las nuevas perspectivas de la didáctica de la Historia de corte más analítico que pone énfasis en una comprensión profunda los fenómenos sociales y que trabaja con sujetos diferentes de los grandes personajes patrios. Esta última perspectiva se encuentra en relación con lo objetivos declarados en el currículum para la asignatura de Historia. Sin embargo, la presencia de la historia tradicional nos muestra una cierta dicotomía entre lo declarado y lo propuesto para que sea trabajado en aula, puesto que los 
OA no necesariamente apuntan al desarrollo del pensamiento histórico ni a la comprensión de la sociedad en la que vive el estudiante, sino más bien al conocimiento de la Historia nacional.

\section{Relaciones entre el currículum de Historia y Ciencias Sociales y el contexto de producción}

Hasta ahora en el análisis del currículum se observa que la enseñanza de la Historia bascula entre perspectivas tradicionales que prioriza un aprendizaje de acontecimientos de forma memorística y las de la Didáctica de las Ciencias Sociales actual, que promueve el pensamiento histórico. Aprehendiendo el currículum desde el materialismo cultural de Raymond Williams, reconocemos que se forjan en un determinado contexto, en nuestro caso aun en desarrollo, caracterizado por la tensión entre los círculos políticos y la sociedad civil que ha concluido con la transformación de la estructura educativa. La derogación de la ley educativa de la dictadura (LOCE) fue la primera materialización de este cambio que no nació desde las cúpulas dirigentes, aun cuando ya en el sistema político institucional había miembros -incluida la presidente Michelle Bacheletque ideológicamente no se encontraban a favor de la estructura política impuesta en los años '70 y ' 80 .

Bajo la premisa de que los productos culturales se articulan a partir de la estructura de sentir (Williams, 2000), la permanencia de una enseñanza de la Historia tradicional en el currículum tendría relación con los fines ideológicos a los que remiten y a determinadas ideas construidas sobre ella -desde la reflexión o no- a partir de la experiencia -individual y colectiva, directa e indirecta. La historia escolar desde su aparición en Chile estuvo marcada por el interés primero de moralizar a los miembros de la nación (Serrano, 2014; Zúñiga González, 2015) y luego para lograr su adhesión a ella (Lira, 2013). Análisis contemporáneos sobre la enseñanza de la Historia en Chile siguen demostrando que los idearios nacionalistas en la escuela tienen gran vigencia (Garrido González, 2009), lo cual coincide con nuestros resultados detallados en el apartado anterior.

Creemos que la continuidad de una enseñanza de la historia tradicional responde en parte a las construcciones históricas de la materia educativa de larga data, o residuales siguiendo a Williams. Esta concepción puede ser explicada por la forma en cómo se ha ideado y enseñado la historia en Chile. Desde el siglo XIX y hasta principios del siglo XX, esta estuvo centrada en hechos y acontecimientos bajo una perspectiva narrativa (Zúñiga González, 2015), tendencia que según Lira (2013) se modificó en los años sesenta, pero que la dictadura militar 
volvió a tornar episódica y nacionalista (Reyes, 2002; Toledo Jofré \& Renato Gazmuri Stein, 2009).

Este recorrido nos muestra una permanencia a pesar de los nuevos requerimientos para la educación señalados en distintas instancias desde el retorno a la democracia. Allí se plantó la necesidad de formar sujetos acorde a las condiciones de la sociedad actual (Comisión Nacional para la Modernización de la Educación, 1995; Consejo Asesor Presidencial para la Calidad de la Educación, 2006). Una educación histórica basada en la mera repetición de acontecimientos políticos de la Historia de Chile en base a la memorización no se ajustaría al tiempo presente, puesto que "por sobre los contenidos cobran importancia los procesos formales o conductuales que es necesario para manejar el aprendizaje: el aprender a aprender" (Comisión Nacional para la Modernización de la Educación, 1995: 12). La educación hoy "debe contribuir al logro de una actitud reflexiva y crítica por parte del educando, quien debe aprender a valorar su propia libertad de pensamiento y su juicio autónomo. De este modo, el estudiante podrá jugar un rol activo en la propuesta e implementación de cambios que resulten en un desarrollo mas pleno de sí mismo y de su entorno" (Consejo Asesor Presidencial para la Calidad de la Educación, 2006: 60).

Desde hace veinte años se han hecho declaraciones como las citadas anteriormente. Nuestros resultados indican que, al menos en lo concerniente a la educación básica en la asignatura de Historia, estas intenciones no se logran materializar totalmente, puesto que se sigue promoviendo una historia nacional con baja complejidad cognitiva y un escaso desarrollo del pensamiento histórico. Hayamos su explicación en que los elementos residuales "son (...) vividos y practicados sobre la base de un remanente -cultural tanto como social- de alguna formación o institución social y cultural anterior" (Williams, 2000: 144), puesto que aun tienen su funcionalidad llegando a formar parte de lo dominante.

Los hallazgos de esta investigación no solo muestran la pervivencia de estas formas tradicionales de la enseñanza de la Historia, sino también la creciente incorporación de otras estrategias didácticas y perspectivas históricas. Miradas analíticas, la adquisición de conceptos propios de la ciencia de referencia, el trabajo con fuentes y la incorporación de otros sujetos sociales, son aspectos novedosos en el currículum. Aquí el contexto histórico juega un rol importante. En distintas instancias se ha bogado por cambiar las formas tradicionales de educación (Comisión Nacional para la Modernización de la Educación, 1995; Consejo Asesor Presidencial para la Calidad de la Educación, 2006). El currículum y la ley educativa vigente también lo señalan, argumentando que la enseñanza de la Historia debe contribuir al desarrollo del niño para su desenvolvimiento en la sociedad (democrática). Estas narrativas emergentes implican la generación de nuevos significados y valores (Williams, 2000), los cuales con- 
trastan en este caso con la enseñanza de la Historia tradicional a la que apuntan los objetivos de aprendizaje.

Como sostiene Williams, estos pueden localizarse dentro de lo dominante o bien en contraposición a ello (2000), con un doble lugar de enunciación. El caso aquí analizado presenta estas dos vertientes. Por un lado correlaciona con lo dominante, en este caso institucional, aparecen explícitos en la documentación oficial. Por otro lado, un lugar contrahegemónico, en tanto son requerimientos establecidos también por la sociedad civil, desprovista del poder político hegemónico. Así, por ejemplo, los estudiantes en Chile establecían como uno de los primeros puntos de sus demandas "una reforma a la malla curricular, para formar seres humanos íntegros, con valores y principios necesarios para conformar una sociedad, teniendo una educación consciente del medio ambiente y de las raíces culturales indígenas del país" (CONES, 2011). También el Movimiento por la Historia, la Geografía y las Ciencias Sociales declaró que "no pretendemos formar en el colegio historiadores, geógrafos ni cientistas sociales, no es el propósito, pero sí niños y niñas que lean historia, que conozcan documentos, que sean capaces de interpretar, y en esa capacidad de interpretación tengan la posibilidad de sopesar las formas de la escritura y la lectura. También importa que se reconozcan como parte de un espacio social y culturalmente intervenido, asociado a identidades locales, nacionales y globales y con conciencia de cuál es la relación entre ellos y ellas y el territorio que habitan" (Movimiento por la Historia, la Geografía y las Ciencias Sociales, 2010: 43).

En estos movimientos sociales, sus discursos y sus demandas en favor de una enseñanza alejada de la repetición de contenidos, centrada en la formación de sujetos activos aparecen en un determinado contexto, en última instancia, coyuntural y emergente. La movilización del año 2006 comenzó por una cuestión muy concreta, el alza en el arancel de la Prueba de Selección Universitaria, que prontamente derivó en cuestionamientos a la estructura del sistema educativo y tuvo su continuidad en el movimiento de 2011. Como en este caso, el movimiento de 2010 de Historiadores y profesorado emergió como respuesta al intento de reducir las horas lectivas de la asignatura.

En ambos casos las críticas a la forma de enseñanza de la Historia son secundarias, pero forman parte de un proyecto global contrahegemónico para la educación chilena. Así, estas construcciones emergentes adquieren potencia suficiente en un contexto de crisis política, en el que se ha puesto en jaque el modelo ideológico, económico y político (Mayol, 2013). En este sentido la incorporación de nuevos sujetos sociales, y la inclusión de perspectivas didácticas contemporáneas en la parte introductoria del currículum, que favorecerían la formación de sujetos críticos y activos, responde a estas fuerzas emergentes. 
La pervivencia de formas tradicionales y la inserción de perspectivas contemporáneas convergen en lo que Williams denomina dominante, en tanto su área de penetración efectiva es mucho mas amplia que la residual y la emergente (2000). Son las selecciones, las que finalmente imperan en las prácticas culturales, en nuestro caso la enseñanza de la Historia propuesta en el currículum. Estas tienen un alto alcance social, ya que se espera sean trabajadas en las aulas chilenas. Consideramos que no es arbitraria la elección de mantener enclaves puntuales de la historiografía nacional conservadora en momentos asociados a la construcción de los relatos nacionales, sino que tiene relación con preservar ciertos reductos para continuar promoviendo discursos nacionalistas.

Uno de los objetivos explícitos de la educación básica es valorar "la pertenencia a la nación chilena" (Ley General de Educación, 2009: Artículo 29, número 2, letra g.) que aparece como una continuidad de la ley educativa de la dictadura (LOCE), en la cual se establecía que se pretendía "desarrollar su sentido patrio" (Ministerio de Ministerio de Educación, 1990: Artículo 11, letra c.). La ley educativa es el marco jurídico sobre el que se sustenta el sistema educativo, como delimitación de la cultura que se va a transmitir (Apple, 1991). La presencia de la idea de nación chilena con el sentido patrio presente en la ley, así como algunos objetivos de aprendizaje -bajo las perspectivas tradicionales de enseñanza, que no proponen una actitud crítica frente al conocimiento-, corresponderían a las fuerzas dominantes definida por Williams.

De esta manera, el currículum puede ser aprehendido como crisol de las tres dimensiones que intervienen en la articulación de los productos culturales. Nuestros resultados se explican por estos tres elementos, los de larga data, los coyunturales y los hegemónicos, cada uno vinculado a su lugar específico de enunciación.

\section{Conclusiones}

El análisis del apartado de Historia del currículum de educación básica bajo criterios didácticos y pedagógicos indica que existe una dicotomía entre lo declarado en la parte introductoria y lo propuesto en los objetivos de aprendizaje, basada en la complementariedad entre enseñanza tradicional y las perspectivas contemporáneas de la didáctica de la Historia.

Esta doble perspectiva concuerda con la bipolaridad en el sistema institucional derivado del contexto histórico en el que convergen fuerzas hegemónicas - institucionales- y contrahegemónicas - de la sociedad civil. De esta manera las demandas sociales han logrado calar, aunque parcialmente, en la construcción de 
los objetivos educativos de Chile. Por tanto el impacto de los movimientos sociales en el país, al menos en lo concerniente a la educación, es intenso y las decisiones políticas no los han dejado al margen.

\section{Referencias bibliográficas}

Anderson L., \& Krathwohl, D. (2001). A Taxonomy for Learning, Teaching, and Assessing: A revision of Bloom's Taxonomy of Educational Objectives. New York.

Apple M. (1991). Ideología y Currículo, Madrid.

Bellatti I., Gámez Ceruelo, V., Trepat Carbonell, C. (2015). Los contenidos curriculares de historia en los países iberoamericanos: una aproximación cuantitativa. [En:] J. Prats, R. Valls, P. Miralles (eds.). Iberoamérica en las Aulas: Qué Estudia y qué Sabe el Alumnado de Educación Secundaria. Lleida, p. 21-100.

Cabalin C. (2012). Neoliberal education and student movements in Chile: inequalities and malaise. "Policy Futures in Education" Vol. 10, no 2, p. 219-228.

Carrasco Segura W. (2007). Chile, de la Revolución Pingüina a la Ley General de Educación. Un intento de política pública bajo el enfoque de derechos, Santiago de Chile.

Carretero M. (2011). Comprensión y aprendizaje de la historia. [En:] Enseñanza y aprendizaje de la historia en la Educación Básica, Cauhtémoc, p. 69-104.

Comisión Nacional para la Modernización de la Educación (1995). Los desafíos de la educación chilena frente al siglo XXI. Santiago de Chile.

CONES. (2011). Petitorio Nacional Estudiantes Secundarios (CONES) [Blog]. Recuperado de http://coneschile.blogspot.com.es/p/petitorio-secundario.html (Consulta 15 de septiembre de 2014).

Consejo Asesor Presidencial para la Calidad de la Educación. (2006). Informe final de Consejo Asesor Presidencial para la Calidad de la Educación. Santiago de Chile.

Donoso S. (2004). Reforma y política educacional en Chile. 1990-2004. "Estudios Pedagógicos" Vol. 31, no 1, p. 113-135.

Fink N. (2005). Pupil's Conceptions of History and History Teaching. "International Journal of Historical Learning, Teaching and Research” Vol. 5, $\mathrm{n}^{\circ}$ 1. Recuperado de http://centres. exeter.ac.uk/historyresource/journal9/papers/fink.pdf (Consulta 22 de septiembre de 2015).

Garrido González C.F. (2009). Mi vecino, ¿mi enemigo? Nacionalismo en los textos escolares de historia de educación básica en Chile (1981-2006). [En:] Seminario Internacional: Textos Escolares de Historia y Ciencias Sociales, Santiago de Chile 2008. Santiago de Chile, p. 346-360.

Gazmuri Stein R. (2013). La construcción ideológica del currículum chileno de Historia y Ciencias Sociales, Tesis doctoral no publicada. Barcelona.

Ley General de Educación (2009). Pub. L. No. 20.370.

Lira R. (2013). Formación ciudadana y Cuestión Social: evolución de las asignaturas de Historia $y$ Educación Cívica entre fines del siglo XIX y principios del XX. "Cuadernos Chilenos de Historia de la Educación", n 1, p. 26-63.

Mayol A. (2013). El derrumbe del modelo: la crisis de la economía de mercado en el Chile contemporáneo. Santiago de Chile.

Ministerio de Educación, Bases curriculares 2012. Santiago de Chile. 
Ministerio de Ministerio de Educación, Ley Orgánica Constitucional de Enseñanza. (1990). Pub. L. No. 18.962.

Movimiento por la Historia, la Geografía y las Ciencias Sociales, Reducción de Horas en Ciencias Sociales y Tecnología. Los desconocidos argumentos pedagógicos del Ministerio de Educación (2010). "Revista Docencia" n 42, p. 40-44.

Prats J. (ed.). (2011). Didáctica de la Geografía y la Historia, Madrid.

Reyes L. (2002). ¿Olvidar para construir Nación? Elaboración de los planes y programas de estudio de Historia y Ciencia Sociales en el período post-autoritario. "Cyber Humanitatis", no 23, Recuperado de http://www.cyberhumanitatis.uchile.cl/index.php/RCH/article/viewArticle/5606/5474 (Consulta 20 de mayo de 2015).

Rüsen J. (2004). Historical consciouness: narrative structure, moral function and ontogenic development. [En:] P. Seixas (ed.). Theorizing Historical Consciousness. Toronto, p. 63-85.

Sáez-Rosenkranz I. (2016). La metodología histórica aplicada a la investigación educativa. "REIRE. Revista d'Innovació i Recerca en Educació”, Vol 9, no 2, En prensa. Recuperado de http://revistes.ub.edu/index.php/REIRE/article/view/15249 (Consulta 03 de abril 2016).

Sáiz Serrano J. (2013) Alfabetización histórica y competencias básicas en libros de texto de historia y en aprendizajes de estudiantes. "Didáctica de las Ciencias Experimentales y Sociales" no 27, p. 43-66.

Salazar-Jiménez R., Barriga-Ubed, E., Ametller-López, Á. (2015). El aula como laboratorio de análisis histórico: el nacimiento del facismo en Europa. "REIRE. Revista d'Innovació i Recerca en Educació" Vol. 8, no 2, p. 94-115. http://doi.org/10.1344/reire2015.8.2826

Seixas P., Morton T. (2013). The Big Six Historical Thinking Concepts. Toronto.

Serrano S. (2014). Enseñanza de la historia e identidad nacional: Un vínculo a historizar desde la experiencia chilena, 1850-1930. "Encounters/Encuentros/Rencontres on Education" no 15, p. 209-222.

Toledo Jofré M.I., Gazmuri Stein R. (2009). Obedientes memoriones o reflexivos pensantes: tensiones entre objetivos identitarios y cognitivos en enseñanza de la Historia reciente de Chile en $6^{\circ}$ año de enseñanza básica. "Estudios Pedagógicos” Vol. 35, n 2, p. 155-172.

Trepat C.A. (1995). Procedimientos en Historia. Un punto de vista didáctico. Barcelona.

Williams R. (1981). Sociología de la cultura, Barcelona.

Williams R. (1983). Keywords. A vocabulary of culture and society. New York.

Williams R. (2000). Marxismo y literatura (2a. edición), Barcelona.

Wineburg S.S. (2001). Historical Thinking and Other Unnatural Acts: Charting the Future of Teaching the Past. Philadelphia.

Zúñiga González C.G. (2015). ¿Cómo se ha enseñado historia en Chile? Análisis de programas de estudio para enseñanza secundaria. "Pensamiento Educativo: Revista de Investigación Educacional Latinoamericana” Vol. 52. n² 2, p. 119-135. http://doi.org/10.7764/PEL.52.1.2015.9

Zúñiga-Peña J.-M. (2015). El sistema educativo chileno y el fracaso del paradigma neoliberal. "REIRE. Revista d'Innovació i Recerca en Educació" Vol. 8, n 1, p. 67-91. 